\title{
SIMULINK MODEL FOR AUTOMATIC DETECTION AND COUNTING OF THE NUMBER OF WHITE-FLIES ON PLANTS USING IMAGE PROCESSING
}

\author{
Srikant Sourabha Prusty ${ }^{1}$, K.Parvathi ${ }^{2}$ \\ ${ }^{1} P G$ scholar, School of Electronics Engineering, KIIT University, Odisha, India \\ ${ }^{2}$ Professor, School of Electronics Engineering, KIIT University, Odisha, India
}

\begin{abstract}
The whitefly is a small white insect which feeds from the sap of different variety of plants and causes wide spread destruction. It is one of the most harmful pests for crops like tomato, cabbage, broccoli etc. The small size of the pest, which is around $2 \mathrm{~mm}$ in length, poses a great challenge to detect these pests using image processing technique in the presence of noise, which could be in the forms of leaf veins, trichomes, water droplets and dust etc. Early detection of pests is required to reduce or prevent the damage caused by the white-flies. It is also important to count the number of pests as accurately as possible, because based on the number of pests the amount of pesticide can be determined or appropriate advice can be given to the persons concerned. Manual counting of white-flies is very tedious and error prone if the number of pests is very high. We can do the counting satisfactorily by using efficient image processing methods. In this paper a method is proposed to detect and count the number of white-flies using image processing on Simulink and Matlab software.
\end{abstract}

Keywords:Image Processing, Simulink, whitefly, count

\section{INTRODUCTION}

Agriculture sector plays an important role in a country's economy. But a significant part of the agriculture products gets destroyed or affected by bio-aggressors. The whitefly is one of them. It is estimated that there are more than 1500 identified species of white-flies. Adult White-flies are just over $1 \mathrm{~mm}$ in length. Due to their small size early detection of pests on plants is a very challenging task.

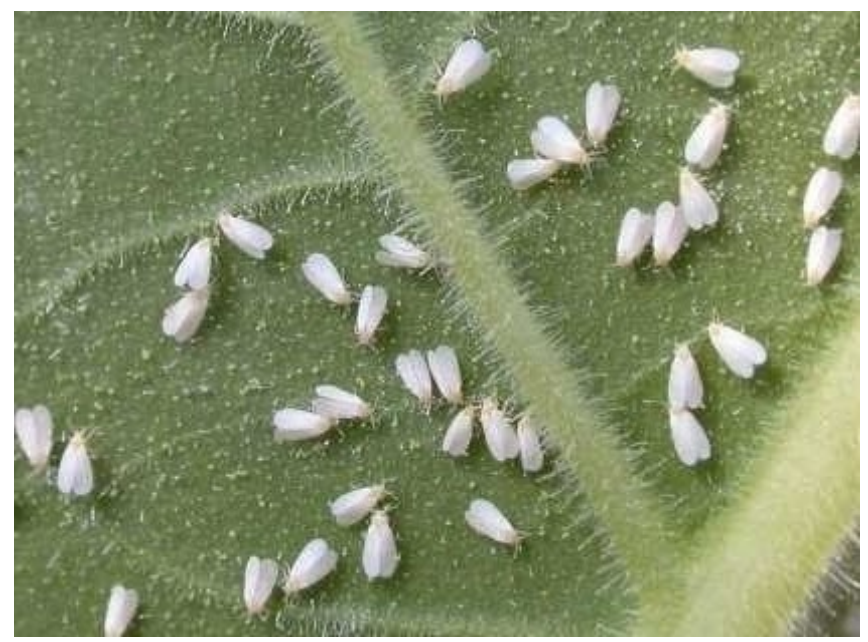

Fig-1 : White-flies

Counting the number of white-flies manually is very tedious and error prone if the number of pests is very high. We can do this satisfactorily by using efficient image processing methods. In this proposed methodology we use a simple image processing technique with the help of Simulink and
Matlab to detect the white-flies and counting their numbers. In 2007, J.Cho and J.Choi proposed a methodology which considers features like size, morphological feature (shape of boundary) and colour components and uses these to identify three kinds of adult insects namely whiteflies, aphids and thrip [10].There is a image processing method based on cognitive vision system that uses knowledge-based techniques to detect mature stage of white fly and count the number of flies on a single leaflet [7].Video based analysis used for detection to detect white flies and aphids which was able to detect eggs of white flies thus analysing behaviour of white flies [8]. There is a method of pest detection and locating 3D positioning of pests based on binocular stereo which was further used to guide a robot to spray pesticides automatically [6].A novel algorithm was proposed which uses relative difference in pixel intensities (RDI) for detecting white fly with an accuracy of $96 \%$ [5].There are also proposed pest detection methods based on clustering [4], extended region based growing [1] ,based on a special neural network, the random subspace classifier (RSC) [2] etc.

\section{PROPOSED METHODOLOGY}

The proposed methodology can be divided into fivesimple steps. 


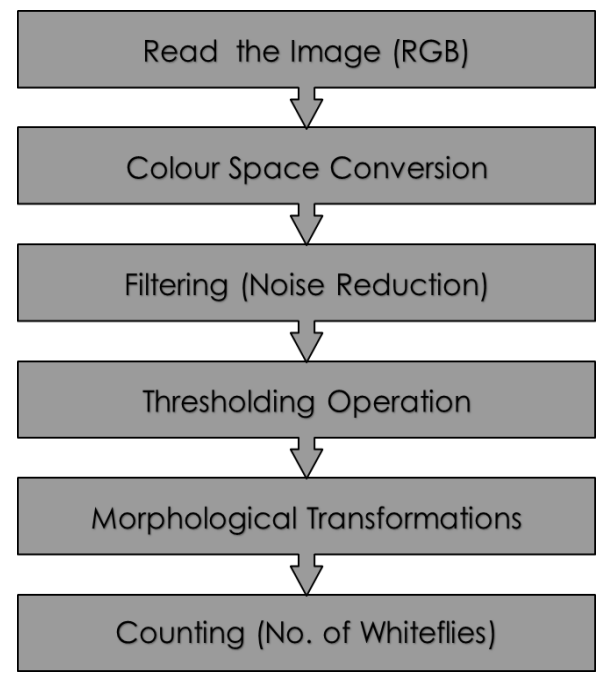

The Simulink model, which is based on these steps with five subsystems, is described below.

\subsection{Colour System Subsystem}

The image database, on which the algorithm is applied, contains RGB colour images .In this Section, basically the RGB colour image is converted into gray scale image after performing smoothing operation on the image.

Fig-2: The steps

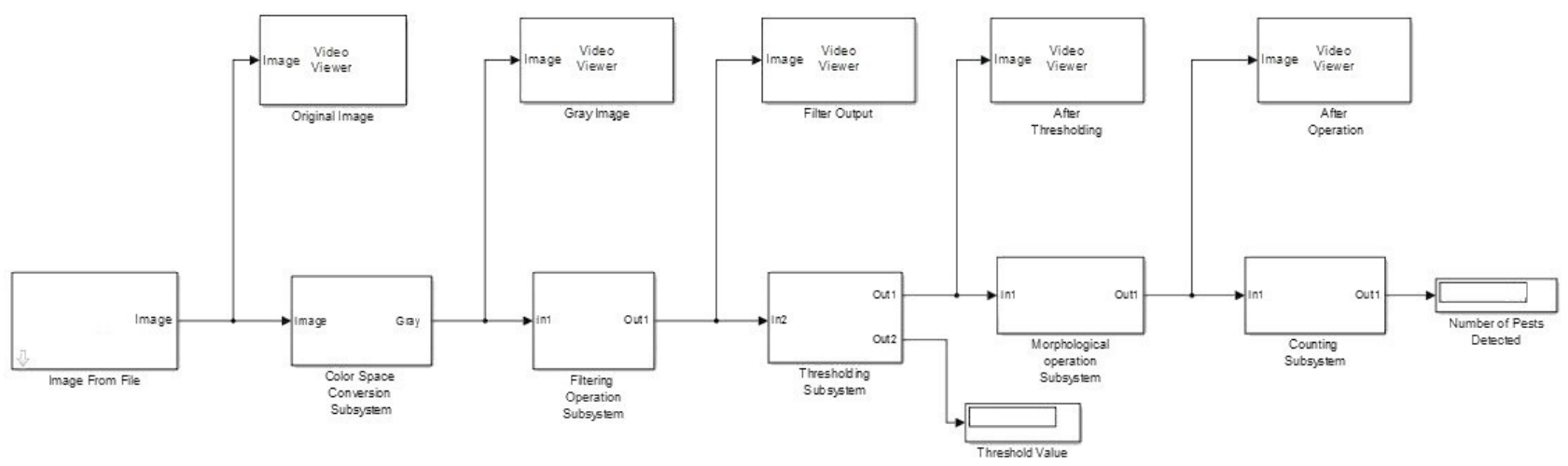

Fig - 3: The Simulink Model

The Gaussian filter and averaging filter is used for image smoothing purpose. By the image smoothing operation the effect of the trichomes (hairy like structures on the leaf) can be reduced. Then Gamma transformation is done to reduce the effect of veins, which have comparable intensity to that of the whiteflies. Then an appropriate color channel is chosen for further processing of the image. The image is then converted to gray scale image to reduce computational complexity.

\subsection{Filtering Subsystem}

In addition to the trichomes, veins of the leaf, noise can be found in the form of water droplets, dusts etc. So, Processing on the image is done to reduce the presence of noise further. Here median filter is for this purpose.

\subsection{Thresholding Subsystem}

Thresholding is required to discriminate foreground data from background data by selecting a suitable threshold value. A global thresholding method called Otsu's thresholdingmethod [11] is used here for this reason.

\subsection{Morphological Operations}

After thresholding operation, it is possible that noise may be present. So for further reduction of noise erosion and dilation process is carried out by selecting a suitable structural element. Generally the objects in the image shrink after the erosion process, to enhance the clarity of the image dilation process is carried out. It might be possible that pests are touching to each other so watershed algorithm is used, but the disadvantage is that it sometimes produces over segmentation.

\subsection{Counting Subsystem}

For counting the number of connected-components we have used the 8-connectivity approach.

\section{RESULTS \& DISCUSSION}

The Images after different stages are shown below. 


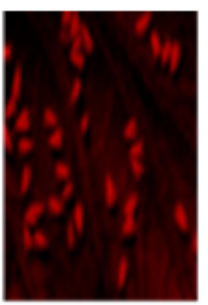

(a)

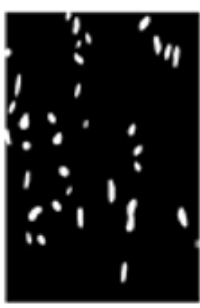

(e)

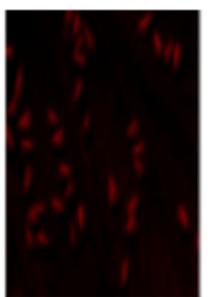

(b)

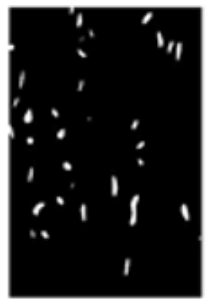

(f)

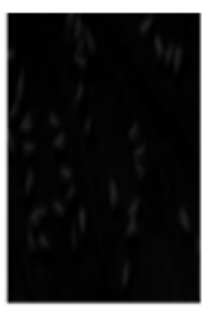

(c)

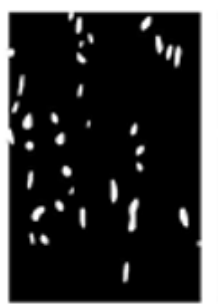

(g)

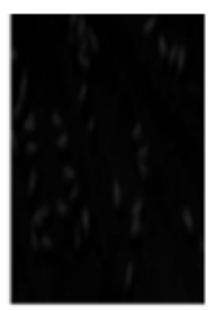

(d)

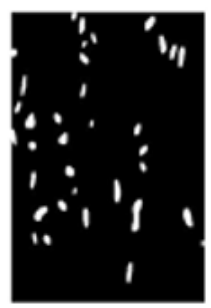

(h)
Fig -4:Image at different Stages of Processing of image -3 of image database (fig.5)

Fig-4(a): R-channel after smoothing operation

Fig-4(b): Gamma Correction

Fig-4(c): Gray Image

Fig-4(d): Filter Output

Fig-4(e): Image after Thresholding

Fig-4(f): Image After erosion

Fig-4(g): Image After dilation

Fig-4(h): Final Image
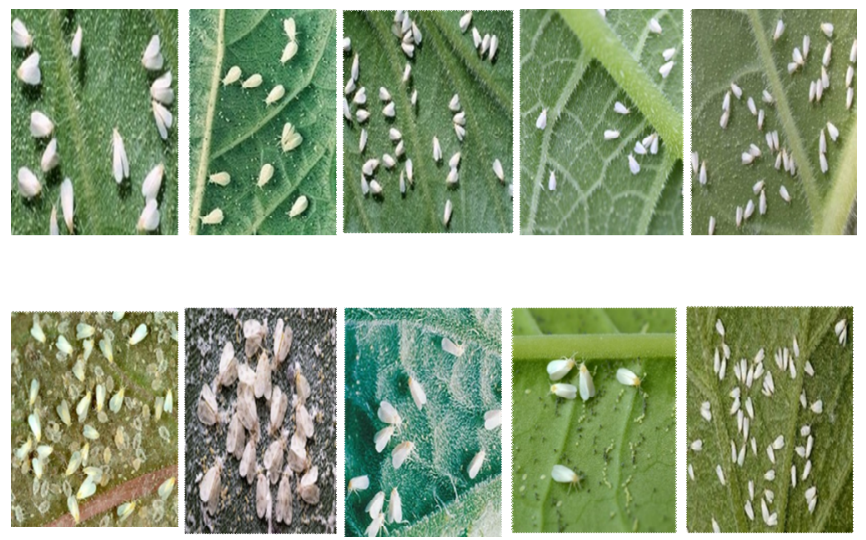

Fig 5: Image Database(Source: Internet)

Table -1: Name of the Table

\begin{tabular}{|l|l|l|}
\hline Sl No. & $\begin{array}{c}\text { Actual number of pests } \\
\text { on leaf (Manual } \\
\text { counting) }\end{array}$ & $\begin{array}{c}\text { Automatic } \\
\text { counting by } \\
\text { Simulink Program }\end{array}$ \\
\hline 1 & 13 & 13 \\
\hline 2 & 11 & 12 \\
\hline 3 & 39 & 36 \\
\hline 4 & 12 & 12 \\
\hline 5 & 36 & 35 \\
\hline 6 & 27 & 28 \\
\hline 7 & 18 & 32 \\
\hline 8 & 11 & 17 \\
\hline 9 & 5 & 5 \\
\hline 10 & 51 & 51 \\
\hline
\end{tabular}

As we can see from the result (table-1,S1 No-7) if the pests are touching or overlapping each other very much and there are white spots (intensity comparable to that of the whitefly) on the background as noise then the percentage of error may be more .

\section{CONCLUSIONS}

Simulink model is developed to detect and count the whiteflies on plants. The model has been tested with database of ten images (Figure 5). The number of whiteflies also verified with manual count. However except two images the model is given the satisfactory results. The overlap of the whiteflies is giving a duplicating the number which resulting double count of the flies. Model may be further modify to overcome of these problems. The hardware implementation may pave the path to chip design which can be installed in mobile phones or any handheld devices easily handled by Farmers.

\section{REFERENCES}

[1] A. Martin, D.Sathish, C.Balachander, T.Hariprasath, G.Krishnamoorthi, Identification and Counting of Pests using Extended Region Grow Algo- rithm, IEEE sponsored 2nd International conference on Electronics and communication (ICECS), 2015.

[2] Lucero Roldn-Serrato, Alberto Escalante-Estrada , Recognition of Pests on Crops with a Random Subspace Classifier , International Work Conference on Bio-inspired Intelligence (IWOBI) , 2015

[3] Ganesh Bhadane, Sapana Sharma and Vijay B. Nerkar, Early Pest Identification in Agricultural Crops using Image Processing Techniques, International Journal of Electrical, Electronics and Computer Engineer- ing,2013

[4] Murali Krishnan , Jabert .G , Pest Control in Agricultural Plantations Us- ing Image Processing, IOSR Journal of Electronics and Communication Engineering (IOSR-JECE) , 2013

[5] Sushma R. Huddar, Swarna Gowri, Keerthana K., Vasanthi S.and Sudhir Rao Rupanagudi Novel Algorithm for Segmentation and Automatic Identification of Pests on Plants using Image Processing , IEEE , 2012

[6] Yan Li Chunlei Xia Jangmyung Lee Vision-based Pest Detection and Automatic Spray of Greenhouse Plant IEEE international Symposium on industrial Electronics (ISiE), pp. 920-925, July 2009.

[7] Paul Boissard, Vincent Martin, Sabine Moisan A Cognitive Vision Approach to Early Pest Detection in Greenhouse Crops Computer and Electronics in Agriculture Journal, 62(2):8393, April 2008.

[8] Vincent Martin and Sabine Moisan Early Pest Detection in GreenhousesInternational Conference on Pattern Recognition, December 2008

[9] Vincent Martin, Sabine Moisan, Bruno Paris, and Olivier Nicolas towards a Video Camera Network for Early Pest Detection in Greenhouses. ENDURE International Conference, La Grande Motte France Oct. 2008

[10] Jongman Cho, Junghyeon Choi Automatic identification of whiteflies, aphids and thrips in greenhouse 
based on image analysis International journal of mathematics and computers in simulation March 27, 2007.

[11] Nobuyuki Otsu , " A Threshold Selection Method from Gray-Level Histograms",IEEE TRANSACTIONS ON SYSTEMS, MAN, AND CYBER- NETICS, JANUARY 1979.

\section{BIOGRAPHIES}

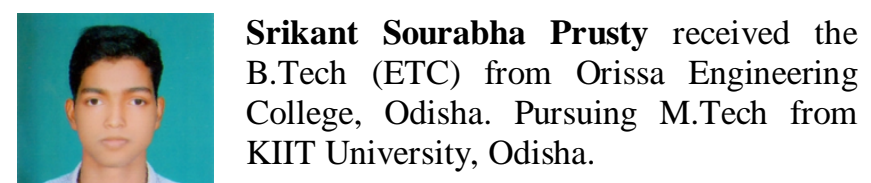
College, Odisha. Pursuing M.Tech from KIIT University, Odisha.

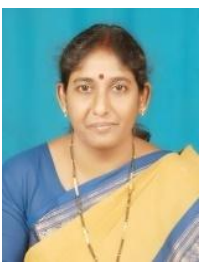

K.Parvathi received the B.E (ECE) from Osmania university. M.Tech \& PhD from Andhra University, AP. Research interests are signal \& Image Processing \& Computational Intelligence. 